\title{
Adhesion analysis of silane coupling agent/copper interface with density functional theory
}

\author{
Mariko MIYAZAKI*, Yoshiharu KANEGAE* and Tomio IWASAKI* \\ *Hitachi Research Laboratory, Hitachi, Ltd. \\ 832-2 Horiguchi, Hitachinaka-shi, Ibaraki 312-0034, Japan \\ E-mail: mariko.miyazaki.jm@hitachi.com
}

Received 9 October 2013

\begin{abstract}
Nanotechnology devices with strong adhesion strength are required due to the miniaturization and reduction of the thickness of electronic devices. This paper describes a technique to select a silane coupling agent effective for obtaining the strong adhesion with copper by use of a density functional theory (DFT) in addition to an experimental peel test. We calculated the adhesion energy at the interface between three candidate silane coupling agents, aminoethyl-aminopropyltrimethoxysilane (AEAPS), mercaptopropyltrimethoxysilane (MPS), and aminopropyltrimethoxysilane (APS), and the copper in order to evaluate the adhesion strength at the interface. The adhesion energy obtained from DFT simulations increased in the order of AEAPS/copper > MPS/copper >APS/copper. The peel strength obtained from an experimental peel test increased in the same order as the adhesion energy obtained from the DFT simulation. Thus, AEAPS was selected as an effective coupling agent for obtaining the strong adhesion with copper. The selection method with the DFT simulation in addition to a peel test is considered to be effective for selecting the best material with the highest adhesion strength.
\end{abstract}

Key words : Adhesion strength, Adhesion energy, Density functional theory, Silane coupling agent, Thin film

\section{Introduction}

Nanotechnology devices such as the semiconductor packages, recording media, and optical disks, used in electronic equipment contain a laminate structure comprised of different materials and have interfaces between organic materials and metals. It is difficult to maintain high adhesion strength at the interface between organic materials and metals. Organic-material-derived silane coupling agent is used to improve the adhesion strength. Recently, the problem of adhesive fracture at the interface has been becoming more and more serious due to the miniaturization and reduction of the thickness of electronic equipment. So, selecting a coupling agent effective for obtaining strong adhesion with metals is very important in preventing adhesive fracture at interfaces. Adhesion strength can be measured with a peel strength test (Kinloch, et al., 1994). However, quantitative measurement is difficult because the organic material is soft and the effect of deformation is large (Iwamura, 2007). Therefore, selecting an effective coupling agent only by using experimental data leads to the high risk of wrong material selection, and using a computer simulation to evaluate the adhesion strength of a material is helpful in reducing the risk. So, in this study, a density functional theory (DFT) in addition to an experimental peel test was used to correctly select an effective coupling agent.

Density functional theory (DFT) simulation is a method for calculating the property of the interface at the atomic or molecular scale level (Hohenberg and Kohn, 1964). The DFT simulation is a quantum mechanical modeling method for investigating the electronic structure of many-body systems. On the other hand, a technique of a molecular dynamic simulation for determining the adhesion strength of metal/metal (Iwasaki, 1999, 2000, 2001 and 2004), resin/metal (Iwasaki, 2009), resin/ceramics (Iwasaki, 2010), and resin/resin (Miyazaki, et al., 2012) interfaces has been carried out in the previous papers. Molecular dynamic simulation provides a step-by-step numerical solution for the classical equation of motion. The organic material used as a subject of study in one of these previous papers was a high polymer resin (Miyazaki, et al., 2012), and it was applied thick on a substrate. Therefore, it is important to research the macro-dynamic characteristics of the high polymers at the interface. In contrast, the silane coupling agent used in this 
research is a small molecule and is applied as a thin coat on copper substrate. Therefore, we used the DFT simulation in this study because we think that it is important to investigate the electronic characteristics of the adhesion at the interface. In the DFT simulation, adhesion strength is determined by calculating adhesion energy. Adhesion energy is defined as the difference between the total potential energy of the material-connected state and that of the material-separated state.

In this research, we analyzed the adhesion at the interface between the silane coupling agent and copper by using the DFT simulation. Three kinds of silane coupling agents, N- $\beta$ (aminoethyl) $\gamma$-aminopropyltrimethoxysilane (AEAPS), $\gamma$-mercaptopropyltrimethoxysilane (MPS), and $\gamma$-aminopropyltrimethoxysilane (APS), were used as examples (Nakamura, et al., 2007). Although adhesion formation between the silane coupling agent and copper has not been clarified, the nitrogen atoms of AEPAS or APS and a sulfur atom of MPS have a high affinity with copper atoms and the nitrogen or sulfur atoms strongly adsorb to the copper atoms. It was difficult to make a silane coupling agent/copper interface model when we performed a simulation. So we made it possible to perform adhesion analysis of the silane coupling agent/copper interface by establishing a method for modeling the interface. We selected an effective silane coupling agent by using this adhesion analysis with the DFT simulation in addition to an experimental peel test.

\section{Adhesion analysis at the interface}

Figure 1 shows an example of the laminate structure of a semiconductor device. Organic-material-derived silane coupling agent is used to improve the adhesion strength at the interface between an organic insulation film and copper. We analyzed the adhesion at the interface between the silane coupling agent and copper by using the DFT simulation. We calculated the adhesion energy to evaluate the adhesion strength at the interface. Three kinds of silane coupling agents, which are shown in Table 1, were used as candidate materials. The molecular structures of these silane coupling agents are shown in Fig. 2. MPS has a mercapto group and has one sulfur atom, and AEAPS and APS each have amino groups. APS has one nitrogen atom and AEAPS has two. We calculated the energy optimized structure at the interface between one silane coupling agent and copper, and the adhesion energy to evaluate the adhesion strength by using the DFT simulation. "CASTEP" (Segall, et al, 2002) was used for the DFT simulations. "CASTEP" is a module within "Materials Studio®"(Accelrys, 2005), which is a simulation software from Accelrys, Inc. The characteristic of CASTEP code is that a basis function is expanded by plane wave. We used generalized gradient approximation (GGA) (Perdew, 1985) as the exchange correlation. The PBE (Perdew, et al., 1997) function, which is a basic function of some GGA functions, was used. The ultrasoft pseudopotential (Vanderbilt , 1990)was used in order to reduce the plane wave used for expanding the basis function.
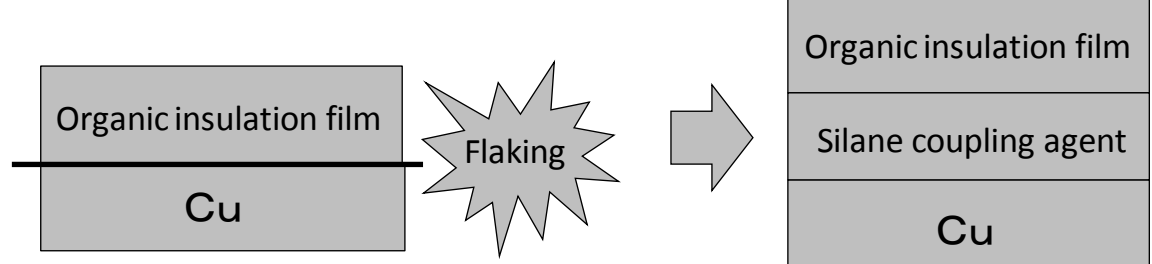

Fig. 1 Example of laminate structure of semiconductor device 
Table 1 Three candidate silane coupling agents

\begin{tabular}{|l|l|}
\hline $\begin{array}{l}\mathrm{N}-\beta \text { (aminoethyl)y -amino } \\
\text { propyltrimethoxysilane } \\
\text { (AEAPS) }\end{array}$ & $\begin{array}{l}\mathrm{NH}_{2}\left(\mathrm{CH}_{2}\right)_{2} \mathrm{NH} \\
\left(\mathrm{CH}_{2}\right)_{3} \mathrm{Si}\left(\mathrm{OCH}_{3}\right)_{3}\end{array}$ \\
\hline $\begin{array}{l}\mathrm{Y} \text {-mercaptopropyltri } \\
\text { methoxysilane (MPS) }\end{array}$ & $\mathrm{HS}\left(\mathrm{CH}_{2}\right)_{3} \mathrm{Si}\left(\mathrm{OCH}_{3}\right)_{3}$ \\
\hline $\begin{array}{l}\mathrm{Y} \text {-aminopropyltri } \\
\text { methoxysilane (APS) }\end{array}$ & $\mathrm{NH}_{2}\left(\mathrm{CH}_{2}\right)_{3} \mathrm{Si}\left(\mathrm{OCH}_{3}\right)_{3}$ \\
\hline
\end{tabular}

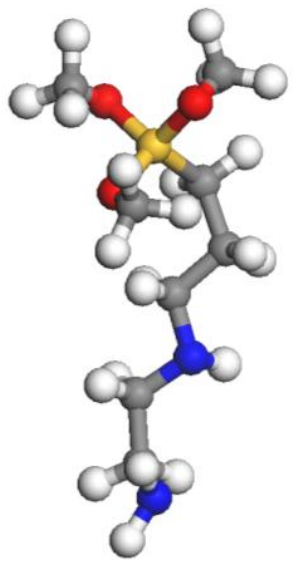

(a) AEAPS

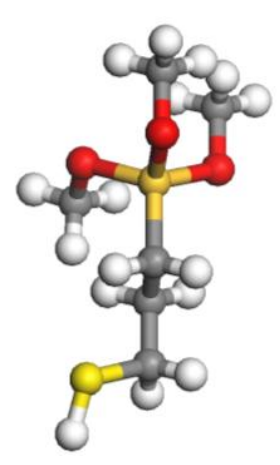

(b) MPS
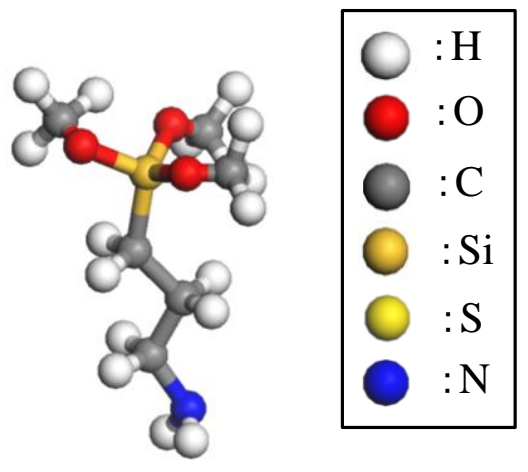

Fig. 2 Molecular structures of silane coupling agents

\section{Modeling method of silane coupling agent/Cu(111) interface}

In an experiment, $\mathrm{a} \mathrm{Cu}(111)$ surface was made by depositing $\mathrm{Cu}$ atoms on a $\mathrm{Cu}$ substrate with electroplating. $\mathrm{By}$ slowing the deposition speed, we can obtain a $\mathrm{Cu}(111)$ surface because the (111) plane of the face-centered-cubic structure has the lower energy than any other surfaces such as the (001) and (110) planes, and because the (111) plane is the most likely to appear on the surface during the slow deposition process. After this process, hydrofluoric acid was used to remove a copper-oxide thin film that was naturally formed with air on the surface. After this cleaning process, the $\mathrm{Cu}(111)$ surface was dipped in an organic solvent that contains a silane coupling agent. While the solvent was evaporated, the coupling agent was stabilized on the $\mathrm{Cu}(111)$ surface. So we use a simulation model that has a silane coupling agent on the $\mathrm{Cu}(111)$ surface.

Figure 3 shows a simulation model. A silane coupling agent is on the vacuum slab of a $\mathrm{Cu}(111)$ surface. The calculation area of the $\mathrm{x}$ and $\mathrm{y}$ directions was $0.767 \mathrm{~nm}$ individually, and that of the $\mathrm{z}$ direction was $0.282 \mathrm{~nm}$. The size of this copper surface model was determined so that the stress in all directions becomes zero. The DFT simulation was performed by the periodic boundary condition. Our object was to obtain an optimized structure of the interface between a silane coupling agent and $\mathrm{Cu}(111)$.

If one silane coupling agent was on $\mathrm{Cu}(111)$ in the initial state, then the silane coupling agent fell flat on the copper layer during the structure optimization, as shown in Fig. 4. However, this is not the most stable state but a locally optimized state. We can get the most stable state, that is, the really optimized state by continuing the structure optimization process for a long time after the locally optimized state shown in Fig. 4 was obtained. Because this process is very time-consuming, we used an efficient modeling method that uses two coupling agent molecules in the first stage, as shown in Fig. 5. We first prepared a vacuum slab of a $\mathrm{Cu}(111)$ surface. The crystal structure of copper is the face-centered cubic structure, and a lattice constant is $0.256 \mathrm{~nm}$. There are three copper atoms in $\mathrm{x}, \mathrm{y}$ and $\mathrm{z}$ direction, respectively. We put two silane coupling agent molecules, and one of the hydrogen atoms bonded to a 
nitrogen or sulfur atom in each coupling agent molecule were deleted, on a $\mathrm{Cu}(111)$ surface (Fig. 5(a)). We then got an optimized structure by the DFT simulation (Fig. 5(b)). This optimized structure is not appropriate for adhesion analysis because large compressive stress in the $\mathrm{x}$ and $\mathrm{y}$ directions exists due to the presence of two coupling agent molecules on the small area. So, we deleted one of two coupling agent molecules (Fig. 5(c)) and optimized the structure by the DFT simulation again. After that, we could get the optimized structure at the interface between the coupling agent molecule and copper (Fig. 5(d)). We finally confirmed that the stress in all directions becomes zero.

The optimized structures from the DFT simulation are shown in Fig. 6. This shows that the nitrogen or sulfur atoms and the copper atoms were close together (the distance between these atoms is about $0.2 \mathrm{~nm}$ ), and it seems that these atoms formed covalent bonds. The number of covalent bonds between the AEAPS and copper was three, while for the MPS and copper or APS and copper, the number of covalent bonds was only two.

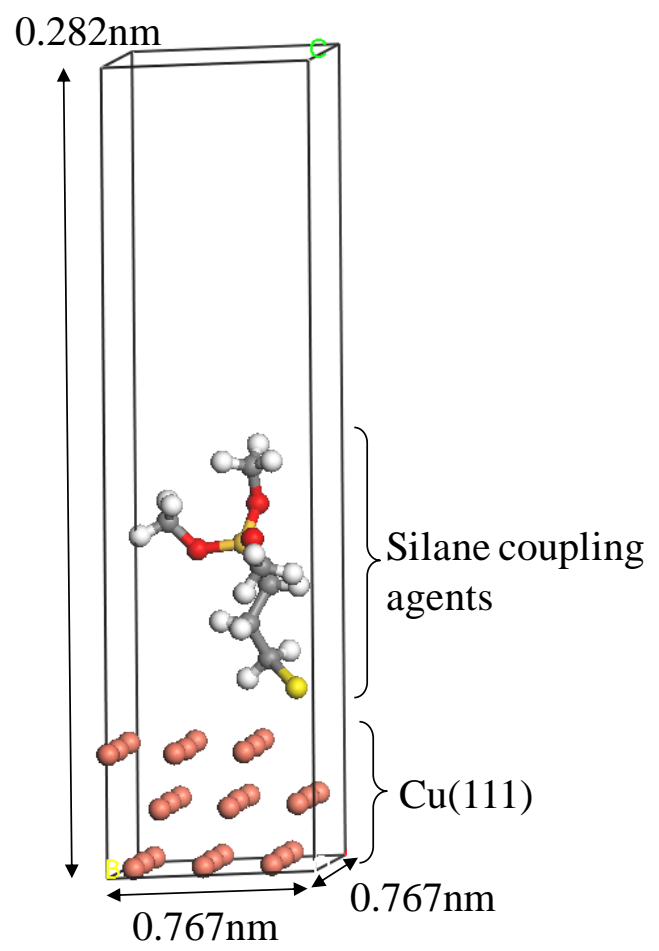

Fig. 3 Simulation model 


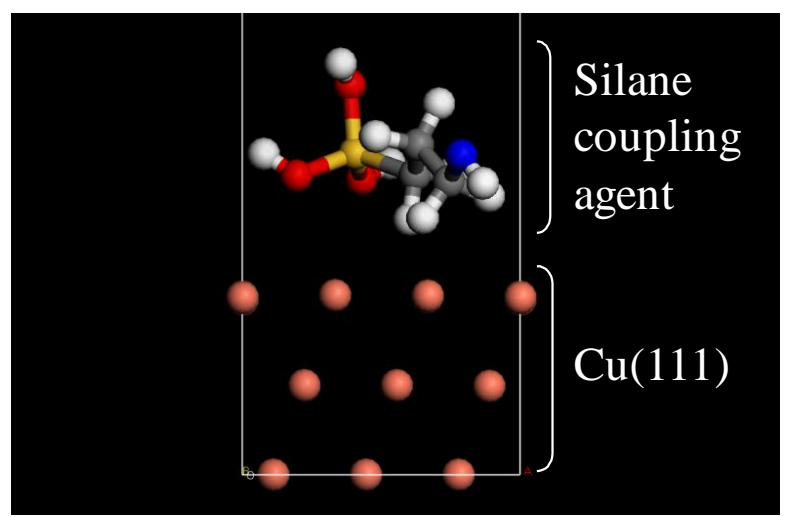

Fig. 4 Locally optimized structure when one silane coupling was used in initial state

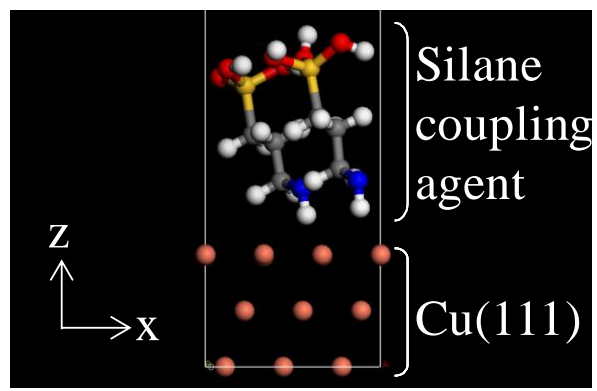

(a) Two silane coupling agents are put on $\mathrm{Cu}(111)$

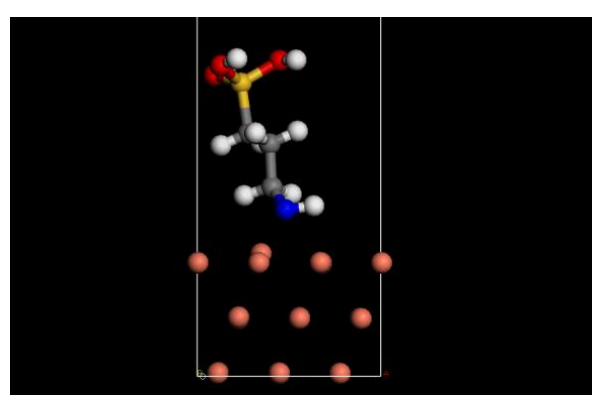

(c) Delete one silane coupling agent

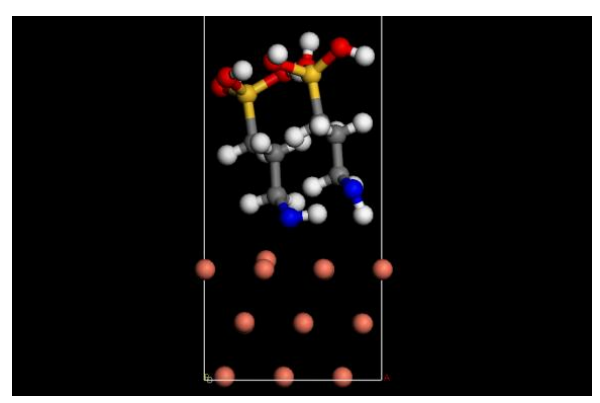

(b) Optimized structure of two silane coupling agents $/ \mathrm{Cu}(111)$ interface

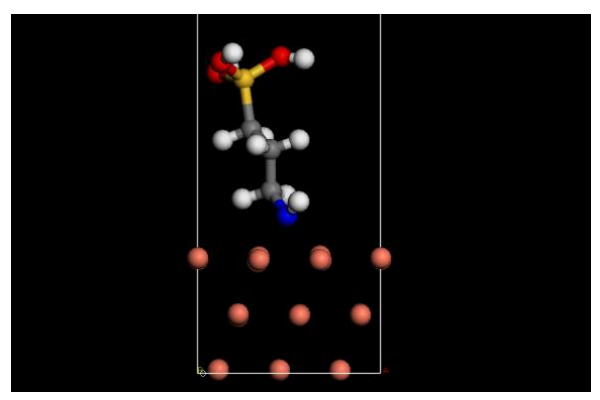

(d) Optimized structure of the silane coupling agent/ $\mathrm{Cu}(111)$ interface

Fig. 5 Modeling method for silane coupling agent/Cu(111) interface 


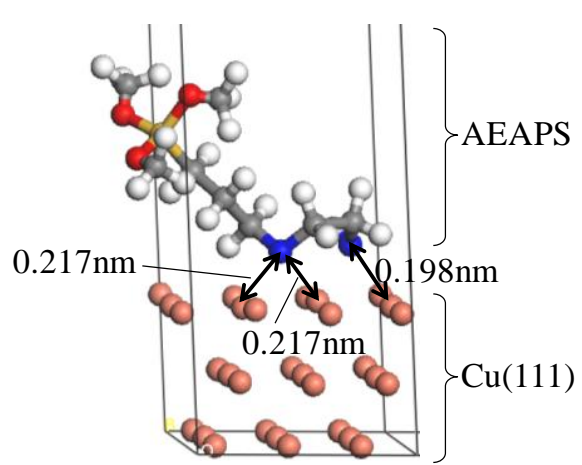

(a) AEAPS/Cu(111)

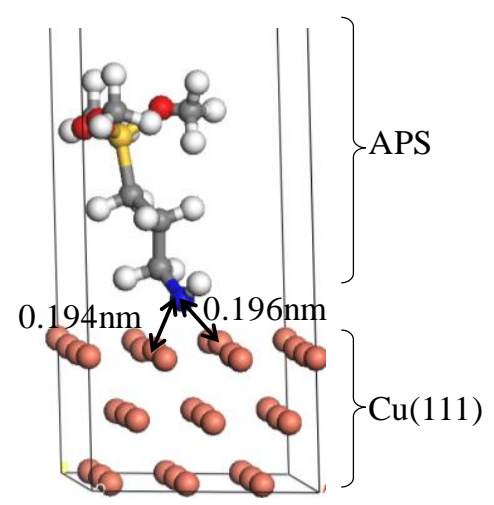

(c) $\mathrm{APS} / \mathrm{Cu}(111)$

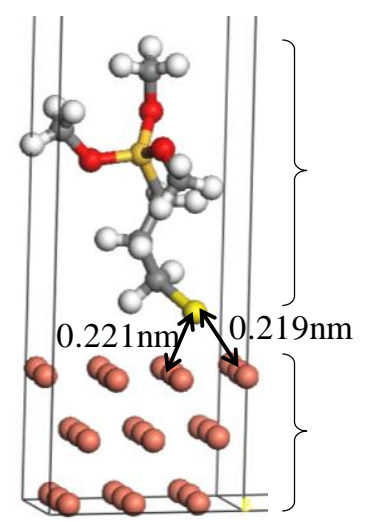

(b) $\mathrm{MPS} / \mathrm{Cu}(111)$

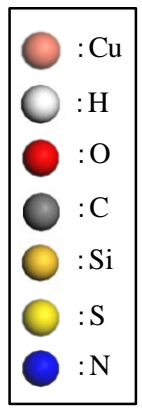

Fig. 6 Optimized structures of silane coupling agent/Cu(111) interface

\section{Results of the adhesion energy}

The adhesion energy between the silane coupling agent and the copper was calculated by using the optimized structures. As shown in Fig. 7, the adhesion energy $\left(E_{a d}\right)$ is defined as the difference between the total potential energy of the material when connected $\left(\mathrm{E}_{\mathrm{co}}\right)$ and when separated $\left(\mathrm{E}_{\mathrm{se}}\right)$ (i.e., $\mathrm{E}_{\mathrm{ad}}=\mathrm{E}_{\mathrm{se}}-\mathrm{E}_{\mathrm{co}}$ ). When the adhesion energy is high, the adhesion strength is high.

The adhesion energy obtained from the DFT simulation and the peel strength measured with the peel strength test are compared in Fig. 8. The peel strength is represented by an arbitrary unit normalized to the quantity of AEAPS in the logarithmic scale. The details of the peel test are shown in chapter 6. Both simulation and experimental results in Fig. 8 show that AEAPS had the highest adhesion strength and that APS had the lowest adhesion strength. As mentioned in chapter 3, there were three covalent bonds between AEAPS and copper, while for MPS/copper or APS/copper, the number of covalent bonds was only two. This seems to explain why AEAPS had stronger adhesion strength than the others. We think that AEAPS has two nitrogen atoms, so it is able to form more covalent bonds. In contrast, MPS or APS have only one sulfur or nitrogen atom, so the number of covalent bonds is less than AEAPS. 


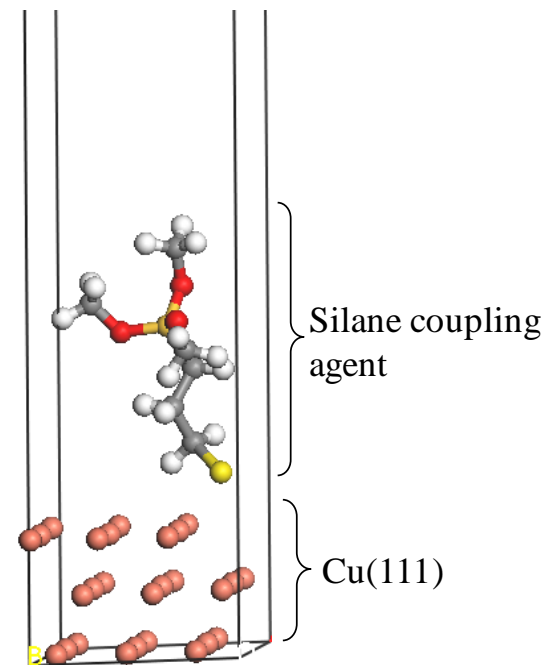

(a) Material-connected state

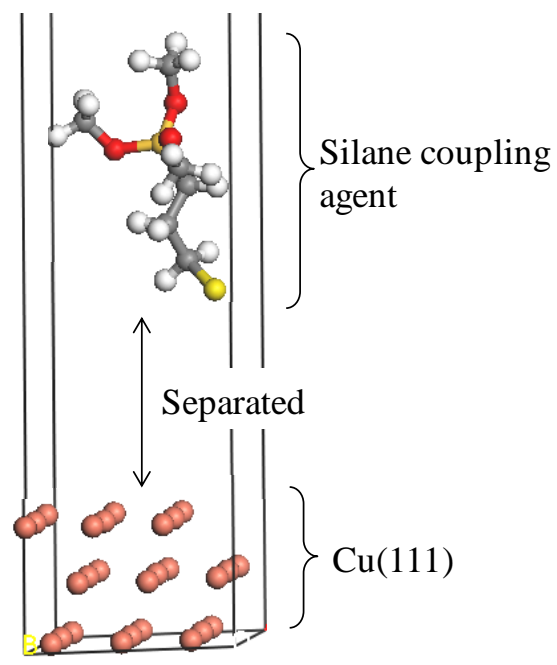

(b) Material-separated state

Fig. 7 Material-connected and material-separated states

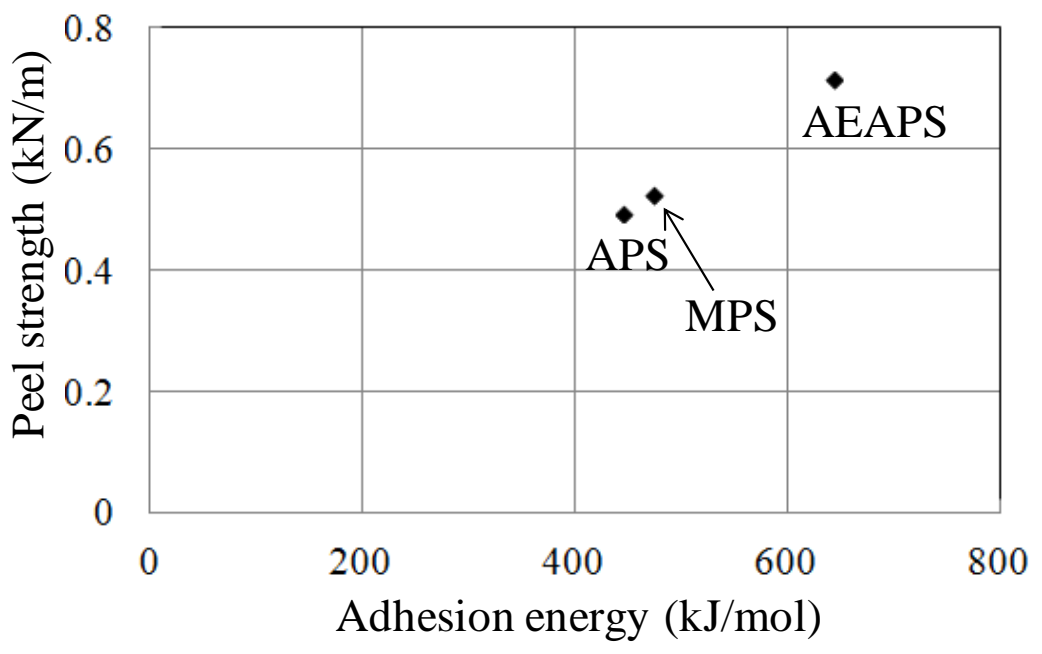

Fig. 8 Results of adhesion energy and peel strength

\section{Results of the electron density distribution at the interface}

The electron density distribution at the interface between the silane coupling agent and copper was calculated to evaluate the number of the covalent bonds. Figure 9 shows pictures of the electron density distribution. These pictures are magnified at the interface. Electron clouds between the silane coupling agents and the copper were bonded, and covalent bonds were formed. As shown in Fig. 9, the number of covalent bonds of AEAPS is three, while the number of covalent bonds between MPS and copper and APS and copper is both two. Because the adhesion energy of AEAPS, which has more covalent bonds with copper than the other coupling agents, is largest of the three candidates, the number of covalent bonds is considered to be a dominant factor in the adhesion energy. Thus, the adhesion energy can be roughly estimated by visually representing the electron density distribution and covalent bonds. 


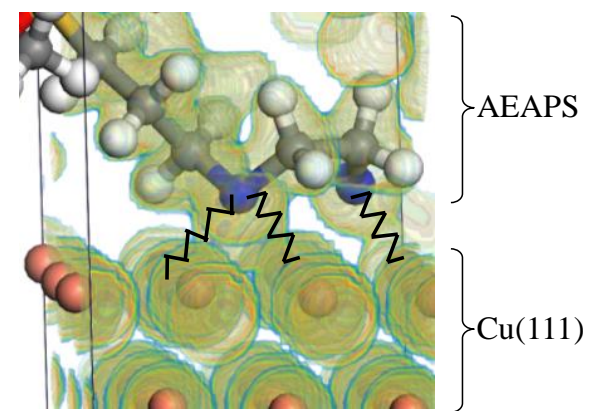

(a) AEAPS/ $\mathrm{Cu}(111)$

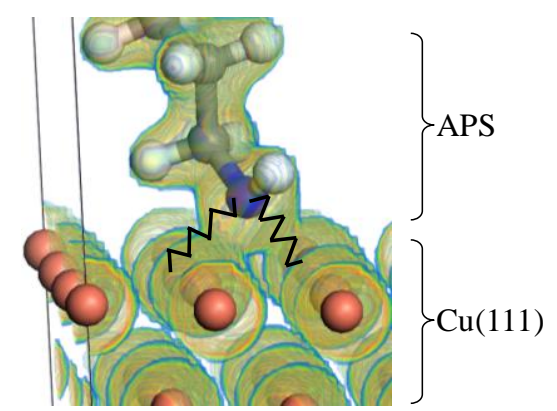

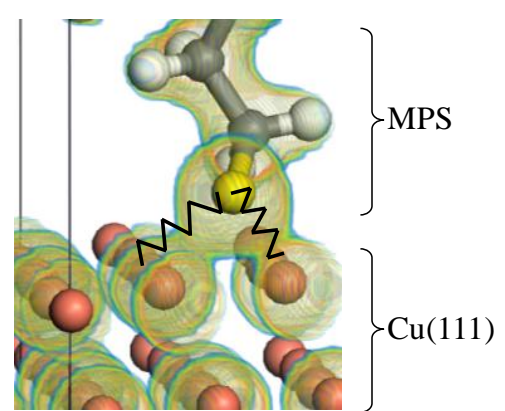

(b) $\mathrm{MPS} / \mathrm{Cu}(111)$

Electron density $\left[\mathrm{e} /(0.1 \mathrm{~nm})^{3}\right]$

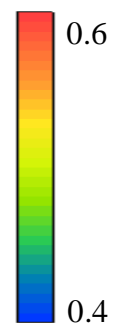

(c) $\mathrm{APS} / \mathrm{Cu}(111)$

Fig. 9 Electron density distribution at interface

\section{Comparison between simulation and experimental results}

To compare the simulation result with an experiment, the peel test was carried out in the following method. Three kinds of silane coupling agents, MPS, APS, and AEAPS, were attached on copper substrates. The thickness of the silane coupling agent was $20 \mu \mathrm{m}$, and that of the copper substrate was $200 \mu \mathrm{m}$. The width and length of the specimens were 1 and $200 \mathrm{~mm}$, respectively. The peeling force was applied to the edge of the silane coupling agent, as shown in Fig. 10, and the silane coupling agent film was pulled away from the copper substrate at a $90^{\circ}$ angle at a peel rate of 50 $\mathrm{mm} / \mathrm{min}$. The peel strength was determined by measuring the peeling force. For each silane coupling agent material, we carried out ten peel tests and averaged the measured results.

The averaged values of the measured peeling forces for the AEAPS, MPS, and APS are shown in Fig. 8. The peel strength obtained from experimental peel tests increased in the same order (AEAPS > MPA > APS) as the adhesion energy obtained from the DFT simulation although the unit of the peel strength is not the same as that of the simulation. So, AEAPS is considered the most effective coupling agent for obtaining the strong adhesion. Thus our method with the DFT simulation in addition to a peel test is considered to be effective for selecting the best material with the highest adhesion strength. 


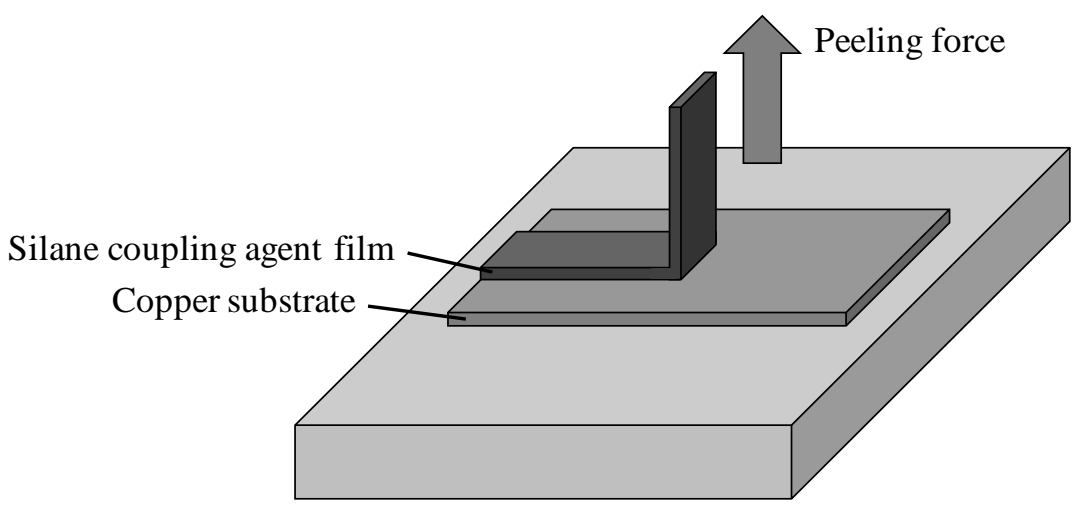

Fig. 10 Schematic view of peel strength test

\section{Conclusions}

To select a silane coupling agent effective for obtaining the strong adhesion with copper, the adhesion at the interface between a silane coupling agent and copper was analyzed by using a DFT simulation, and a peel test was carried out. Three kinds of silane coupling agent, AEAPS, MPS, and APS, were used as examples, and we calculated the adhesion energy between these silane coupling agents and copper to evaluate the adhesion strength at the interface. The peel strength was also measured in the peel test. The following results were obtained.

(1) By using two silane coupling agent molecules in the initial state, we established an efficient method for modeling the silane coupling/copper interfaces, where nitrogen or sulfur atoms of the silane coupling agent adsorb strongly to copper.

(2) The adhesion energy obtained from the DFT simulation showed that the adhesion energy increased in the order of AEAPS/Cu(111) > MPS/Cu(111) > APS/Cu(111).

(3) The peel strength obtained from a peel test increased in the same order as the adhesion energy $(\mathrm{AEAPS} / \mathrm{Cu}(111)>$ $\mathrm{MPS} / \mathrm{Cu}(111)>\mathrm{APS} / \mathrm{Cu}(111))$.

(4) Because AEAPS with high adhesion energy has more covalent bonds between the silane coupling agent and copper than the other two coupling agents, the number of covalent bonds is considered to be a dominant factor in the adhesion energy.

(5) AEAPS was selected as a coupling agent effective for obtaining the strong adhesion to copper by using the DFT simulation in addition to a peel test.

\section{References}

Accelrys, Materials Studio 4.0 (2005).

Hohenberg, P. and Kohn, W., Inhomogeneous electron gas, Physical Review B, Vol. 136 (1964) , pp.864-871.

Iwamura, E., Improvement of adhesion strength in thin films, Journal of the Surface Finishing Society of Japan, Vol. 58, No. 5 (2007) , pp. 260-266 (in Japanese).

Iwasaki, T., Molecular dynamics study of diffusion and atomic configuration in layered structures for Al circuit interconnects, Computational Mechanics, Vol. 24, No. 2 (1999) , pp. 148-154.

Iwasaki, T., Molecular dynamics study of adhesion strength and diffusion at interfaces between interconnect materials and underlay materials, Computational Mechanics, Vol. 25, No. 1 (2000) , pp. 78-86.

Iwasaki, T. and Miura, H., Molecular dynamics analysis of adhesion strength of interfaces between thin films, Journal of Materials Research, Vol. 16, No. 6 (2001), pp. 1789-1794.

Iwasaki, T., Application of molecular-dynamics simulation to interface stabilization in thin-film devices, International Journal of the Japan Society of Mechanical Engineering, Series B, Vol. 47, No. 3 (2004), pp. 470-476.

Iwasaki, T., Molecular dynamics study on the effect of lattice mismatch on adhesion strength between organic materials and metals, Journal of the Society of Materials Science, Japan, Vol. 58, No. 3 (2009) , pp. 257-261.

Iwasaki, T., Molecular dynamics study on the effect of lattice mismatch on adhesion strength between ceramics and organic materials, Journal of the Society of Materials Science, Japan, Vol. 59, No. 2 (2010) , pp. 165-170. 
Kinloch, A. J., Lau, C. C. and Williams, J. G., The peeling of flexible laminates, International Journal of Fracture, Vol. 66 (1994), pp. 45-70.

Miyazaki, M., Kanegae, Y. and Iwasaki, T., Adhesion analysis of resin/resin interface by molecular dynamics simulation, Journal of Solid Mechanics and Materials Engineering, Vol. 6, No.11 (2012), pp.1002-1012.

Nakamura, S., Pavlovic, E. and Edward, J., Fracture energy of epoxy interfaces with layers of different silane coupling agents, Journal of Adhesion, Vol. 83, No. 4 (2007), pp. 351-365.

Perdew, J. P., Accurate Density Functional for the Energy : Real-space cutoff of the gradient expansion for the exchange hole, Physical Review Letters, Vol. 55 (1985), pp.1665-1668.

Perdew, J. P., Burke, K. and Ernzerhof, M., Generalized gradient approximation made simple, Physical Review Letters, Vol. 77, pp. 3865-3868, Vol. 78 (1997), p.1396.

Segall, M. D., Lindan, P. L., Probert, M. I., Pickard, C. J., Hasnip, P. I., Clarke, S. J. and Payne, M. C., First-principles simulation: ideas, illustrations and the CASTEP code, Journal of Physics: Condensed Matter, Vol. 14 No. 11 (2002), pp. 2717-2744.

Vanderbilt, D., Soft self-consistent pseudopotentials in a generalized eigenvalue formalism, Physical Review B, Vol. 41 (1990), pp. 7892-7895. 